\title{
Strain Measurement in Semiconductor Heterostructures by Scanning Transmission Electron Microscopy
}

\author{
Knut Müller, ${ }^{1, \star, \dagger}$ Andreas Rosenauer, ${ }^{1, \star, \dagger}$ Marco Schowalter, ${ }^{1}$ Josef Zweck, ${ }^{2}$ Rafael Fritz, ${ }^{2}$ \\ and Kerstin Volz ${ }^{3}$ \\ ${ }^{1}$ Institut für Festkörperphysik, Universität Bremen, Otto-Hahn-Allee 1, 28359 Bremen, Germany \\ ${ }^{2}$ Institut für Experimentelle und Angewandte Physik, Universität Regensburg, Universitätsstraße 31, \\ 93040 Regensburg, Germany \\ ${ }^{3}$ Materials Science Center and Faculty of Physics, Philipps Universität Marburg, Hans-Meerwein-Straße, \\ 35032 Marburg, Germany
}

\begin{abstract}
This article deals with the measurement of strain in semiconductor heterostructures from convergent beam electron diffraction patterns. In particular, three different algorithms in the field of (circular) pattern recognition are presented that are able to detect diffracted disc positions accurately, from which the strain in growth direction is calculated. Although the three approaches are very different as one is based on edge detection, one on rotational averages, and one on cross correlation with masks, it is found that identical strain profiles result for an $\mathrm{In}_{x} \mathrm{Ga}_{1-x} \mathrm{~N}_{y} \mathrm{As}_{1-y} / \mathrm{GaAs}$ heterostructure consisting of five compressively and tensile strained layers. We achieve a precision of strain measurements of $7-9 \cdot 10^{-4}$ and a spatial resolution of $0.5-0.7 \mathrm{~nm}$ over the whole width of the layer stack which was $350 \mathrm{~nm}$. Being already very applicable to strain measurements in contemporary nanostructures, we additionally suggest future hardware and software designs optimized for fast and direct acquisition of strain distributions, motivated by the present studies.
\end{abstract}

Key words: strain measurement, electron diffraction, TEM, CBED, STEM, semiconductors

\section{INTRODUCTION}

A characteristic of the majority of semiconductor nanostructures is the presence of lattice strain varying at nanometer scale. Originating from the lattice mismatch between layers of different composition deposited during epitaxial growth, strain can appear as a limiting factor for the miscibility of crystals, e.g., GaAs and InAs. Furthermore, compressive strain due to $\mathrm{Ge}_{x} \mathrm{Si}_{1-x}$ regions below the gate electrode in metal oxide semiconductor field effect transistors (MOSFET) is intended to increase the mobility of charge carriers. Consequently, due to its large influence on the physical properties and functionality of devices, accurate and precise measurement of strain in semiconductor heterostructures with high spatial resolution was an active field of research since semiconductor technology was established.

Methods for the measurement of local strain in the field of transmission electron microscopy (TEM) can be divided in two groups-imaging and diffraction. The former originally exploits atomically resolved TEM micrographs with respect to lattice fringe distances (Bierwolf et al., 1993; Bayle et al., 1994; Jouneau et al., 1994; Robertson et al., 1995; Rosenauer et al., 1998). As the observed high-resolution pattern is formed by the interference of all diffracted beams passing the objective aperture, subsequent studies included partly extensive analysis of the phases of

Received December 15, 2011; accepted April 26, 2012

${ }^{\star}$ Corresponding authors. E-mail: mueller@ifp.uni-bremen.de; rosenauer@ifp.unibremen.de

${ }^{\dagger}$ A. Rosenauer and K. Müller contributed to the present work in equal part and share first-authorship.
Bragg beams as a function of specimen thickness, orientation, composition, crystal potential Fourier components, lens aberrations, and defocus (Tillmann et al., 2000; Hÿtch \& Plamann, 2001; Rosenauer et al., 2001; Rosenauer et al., 2006; Guerrero et al., 2007; Müller et al., 2010; Yu \& Mader, 2010). One disadvantage common to all high-resolution TEM techniques is the restricted field of view not only because the lattice fringe pattern must be sampled densely enough to measure fringe spacings precisely, but also because measured fringe distances need to be normalized to (substrate) regions with known strain state, which may be too far away from the region of interest. Dark-field holography methods (Hÿtch et al., 2008; Koch et al., 2010) overcome this problem as they exploit the phase of only one diffracted beam, which nevertheless still depends on many parameters as mentioned above.

In contrast, strain measurement based on diffraction is comprehensively described by the fundamental but simple Bragg law (Bragg \& Bragg, 1913)

$$
2 d_{h k l}(\vec{r}) \sin \theta_{h k l}^{B}(\vec{r})=\lambda
$$

that relates lattice plane spacing $d_{h k l}$ ( $h, k, l$ : Miller Indices), Bragg angle $\theta_{h k l}^{B}$ and wavelength $\lambda$ of the incident radiation that is usually known. Note that both $d$ and $\theta_{h k l}^{B}$ may vary with position $\vec{r}$ in the sample. Considering diffraction into small angles and sufficiently small $\lambda$, the total scattering angle $\theta$ is proportional to the distance $R$ of a Bragg reflection to the primary beam, measured in a recorded diffraction pattern: $2 \sin \theta_{h k l}^{B} \approx 2 \theta_{h k l}^{B}=\theta_{h k l} \sim R_{h k l}$. Thus measuring $R_{h k l}$ locally uniquely yields $d_{h k l}$ and hence lattice strain. 
Taking up the idea of Uesugi et al. (2011), this article addresses three main issues of the scanning convergent beam electron diffraction (CBED) technique: spatial resolution, precision of strain measurement, and future hardware designs optimized for high-speed acquisition of strain maps.

1. Initially experimental details especially on probe formation are given in the following section. Since the diameter of the incident probe is a function of the beam convergence angle, an analysis of settings used in an FEI Titan 80/300 (scanning) TEM (STEM) facility (FEI Company, Hillsboro, OR, USA), whose three-condenser lens system allows for an independent tuning of both beam convergence and beam focus, is included. For GaAsbased semiconductors in [001] projection, we estimate the spatial resolution to be in the range of $0.5 \mathrm{~nm}$, for example.

2. We then report on results of strain measurement by evaluation of CBED disc positions in a highly strained, $350 \mathrm{~nm}$ wide semiconductor heterostructure consisting of a sequence of five $\operatorname{In}_{x} \mathrm{Ga}_{1-x} \mathrm{~N}_{y} \mathrm{As}_{1-y}$ layers with small GaAs buffers in between. The outcome of this section is twofold: On the one hand, we develop three different methods to determine CBED disc positions accurately despite strongly varying intensity distributions inside the CBED discs arising from dynamical interaction of diffracted beams. As a first and most intuitive approach, we fit circles to the edges of discs, obtained by calculating the approximate derivative with the Prewitt operator (Prewitt, 1970). The second method is a real-space method, too, but finds the disc positions by maximizing the difference between annular intensity averages inside and outside a CBED disc. Third, diffracted discs are cross-correlated with different types of masks to calculate relative disc shifts. It is demonstrated that all three methods lead to the same strain profile and exhibit comparable precisions in the range $10^{-4}$ for one single electron beam position. To stress the importance of these studies, we finally compare with results obtained via direct implementation of Uesugi's method (Uesugi et al., 2011). On the other hand, the strain profiles themselves are an important result because the precision belongs to the highest ones the authors are aware of. This is followed by a comprehensive discussion, which in particular shows that cooling and energy filtering are not mandatory for the developed methods to work.

3. Based on the methodical findings of the preceding sections, our concluding section contains concrete future prospects as to possible designs of efficient, precise, and accurate detector hardware suitable for the fast tracking of CBED disc positions.

\section{Materials and Methods}

\section{The Specimen}

The sample contains five ternary and quaternary layers separated by GaAs buffer layers that can clearly be identified

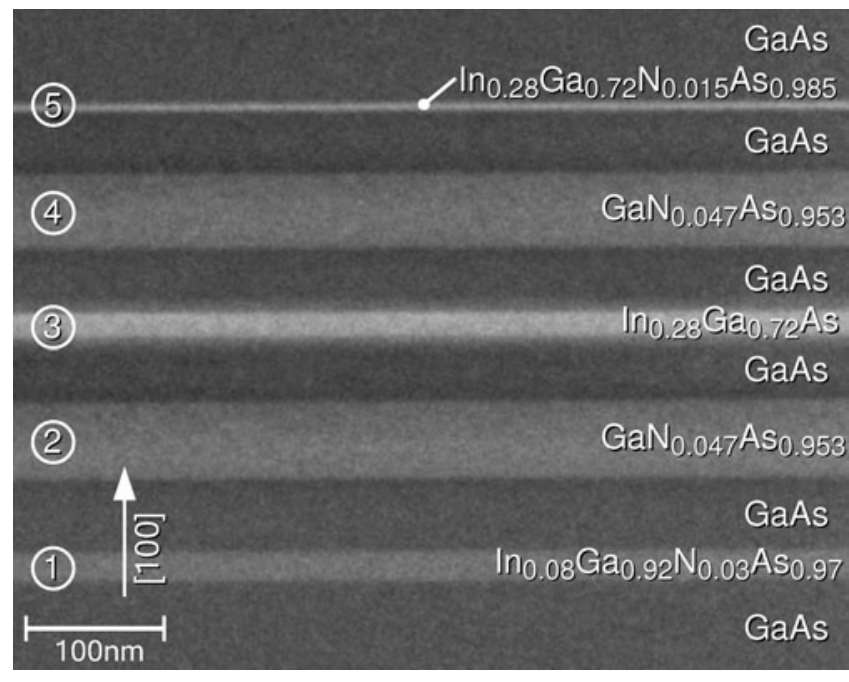

Figure 1. STEM-HAADF image of the sample taken in zone axis [001] with a detector acceptance angle range of 33-200 mrad. Nominal compositions of all five ternary and quaternary layers are given on the right. The sample was grown in [100] direction with layer sequence as shown on the left.

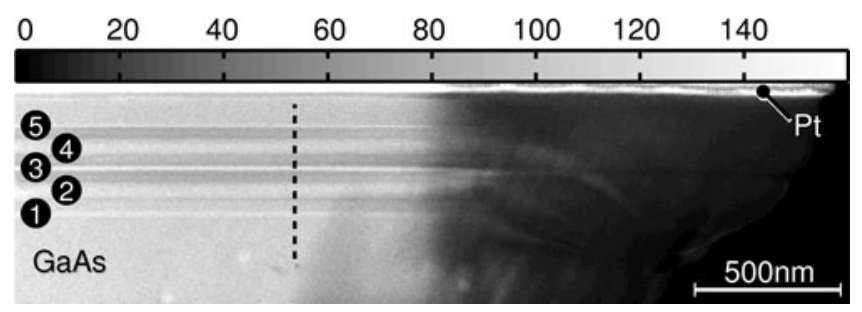

Figure 2. Grayscale thickness map (nm units) of the FIB lamella obtained from HAADF-STEM (Rosenauer et al., 2009). Results are reliable below the Pt cover and outside the indium- and nitrogencontaining layers labeled as in Figure 1. The dashed line indicates the path where the CBED patterns have been acquired.

in the high-angle annular dark-field (HAADF) STEM image in Figure 1. Nominal compositions intended during the metal organic vapor phase epitaxy (MOVPE) are also given and have been derived by high-resolution X-ray diffraction measurements at calibration samples. In the following, we refer to the respective layers using the labeling 1-5 depicted on the left in Figure 1. Growth direction was [100].

A cross-sectional lamella for TEM investigation in [001] zone axis has been prepared by focused ion beam (FIB) etching with a lift-out technique at an FEI Nova 200 NanoLab dual beam facility. Low-energy ion milling at $350 \mathrm{~V}$ and oscillation angles of $\pm 20^{\circ}$ has been performed using a Technoorg Linda (Model GM IV5) facility (Budapest, Hungary) to remove amorphous layers on the specimen surfaces stemming from FIB preparation. Prior to insertion into the microscope, organic material has been removed from the specimen surface by plasma cleaning.

The sample was checked by STEM and high-resolution TEM to ensure the investigated areas depicted in Figure 2 were free of dislocations and stacking defaults. In a dislocation free bulk specimen, strain relaxation only occurs in the 
growth direction, whereas a thin TEM specimen elastically relaxes in the electron beam direction as well. This makes strain measurement in growth direction dependent on specimen thickness for thin specimens. To facilitate a discussion of this effect later, we measured the local specimen thickness by HAADF-STEM as reported recently (Rosenauer et al., 2009). In particular, the HAADF signal recorded with a detector acceptance angle range of 33-200 mrad was normalized to the intensity of the incoming beam and compared with thickness-dependent frozen lattice multislice simulations for pure GaAs carried out with the STEMsim program (Rosenauer \& Schowalter, 2007). This procedure results in the thickness map presented in Figure 2. Note that thickness results are only reliable for unstrained GaAs and neither in the indium and nitrogen containing layers $1-5$, nor in the protective platinum layer deposited during the FIB preparation. CBED patterns were acquired in the vicinity of the dashed line in Fig. 2, where the specimen thickness is approximately $100 \mathrm{~nm}$.

\section{Microscope Condenser Settings}

On the one hand, evaluation of CBED disc positions for strain measurements requires well-separated reflections in the diffraction pattern. On the other hand, spatial resolution of scanning probe techniques suffers from a decrease of the beam convergence due to a cutoff of spatial frequencies by the condenser (usually C2) aperture. It is therefore desirable to set the semiconvergence angle of the incident probe to a dedicated value, which is slightly below the minimum Bragg angle $\theta_{h k l}^{B}$ occuring in a given diffraction geometry. For GaAs projected along [001], this angle is $\theta_{020}^{B}=3.48$ mrad at $300 \mathrm{kV}$ according to equation (1).

The three condenser lens system of an FEI Titan 80/300 (S)TEM facility can be operated in two main modes, TEM and Probe, the former being suited for small and the latter for large convergence angles. Both modes may furthermore be combined with the Microprobe or Nanoprobe setting, which turns the microcondenser lens on or off, respectively. A characteristic of the double zoom provided by the three condenser lenses is that the $\mathrm{C} 2-\mathrm{C} 3$ zoom allows for continuous variation of the beam convergence in ranges defined by an additional option called normal and large angle range. Here, we set the semiconvergence angle of the incident probe to $2.6 \mathrm{mrad}$ using the setting Probe, nanoprobe, large angle range, and a $50 \mu \mathrm{m} \mathrm{C} 2$ aperture. However, the large angle range option causes a strong excitation of the $\mathrm{C} 2$ and C3 lenses, which results in an additional spherical aberration of maximal $0.3 \mathrm{~mm}$, which adds to the usual value of $1.2 \mathrm{~mm}$ for the SuperTwin probe-forming lens in a Titan.

The black curve in Figure 3 depicts the simulated intensity profile of an electron probe imaged onto the specimen with a spherical aberration of $C_{s}=1.5 \mathrm{~mm}$ and a semiconvergence of $\alpha=2.6 \mathrm{mrad}$. In contrast to the gray profile that corresponds to a setting optimized with respect to spatial resolution in an (uncorrected) Titan microscope, we see that the black probe has a diameter comparable to

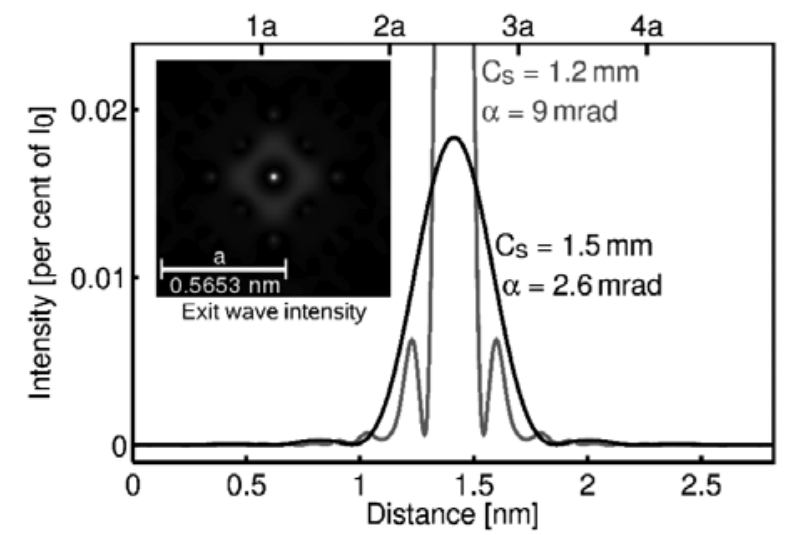

Figure 3. Simulation (Rosenauer \& Schowalter, 2007) of STEM probe intensity profiles for the present experiment (black) and condenser settings optimized for high-resolution STEM (gray) in a Titan microscope operated at $300 \mathrm{kV}$. The axis at the top is scaled in multiples of the GaAs lattice constant $a=0.56533 \mathrm{~nm}$. The inset shows the intensity distribution in the object exit plane computed for a specimen thickness of $100 \mathrm{~nm}$ for illumination along zone axis [001].

the lattice parameter of GaAs. Hence, no atomically resolved strain measurement can be achieved with this probe, but still the spatial resolution is approximately $0.5 \mathrm{~nm}$, measured in terms of the e-length of the black graph in Figure 3. The inset depicts a simulation (Rosenauer \& Schowalter, 2007) of the exit wave intensity at $100 \mathrm{~nm}$ specimen thickness for exact [001] zone axis orientation. The atomic columns are clearly visible as the electrons predominantly channel along them. For this reason, the exit wave intensity is peaked at the projected column positions and decreases in their direct vicinity, so that the GaAs unit cell can be identified. Consequently, the exit wave extension is practically equal to the probe diameter showing a minor influence of beam broadening for such low convergence angles. However, the present experiments have been conducted with a slight mistilt of $0.4^{\circ}$ for reasons that become clear below. In this case, the simulated (Rosenauer \& Schowalter, 2007) exit wave drops to a value of $1 / \mathrm{e}$ at a diameter of $0.7 \mathrm{~nm}$, which was determined from the azimuthally integrated intensity.

\section{Serial CBED Pattern Acquisition}

Series of CBED patterns have been recorded sequentially along a path parallel to the growth direction as shown in Figure 2. The CBED pattern exemplarily depicted in Figure 4 exhibits that essentially one-quarter of the diffraction pattern has been recorded with the primary beam shifted toward the lower left corner. All patterns have been recorded on a Gatan (Pleasonton, CA, USA) UltraScan 2000 charge-coupled device $(\mathrm{CCD}) 2 \mathrm{~K} \times 2 \mathrm{~K}$ camera with a binning of 4 and dwell times of $0.5 \mathrm{~s}$ at each probe position.

Since we aim at a precise recognition of CBED disc edges, a large contrast between CBED reflections and the diffuse background of the diffraction pattern is desirable. This has been achieved in three ways. First, the excitation of 


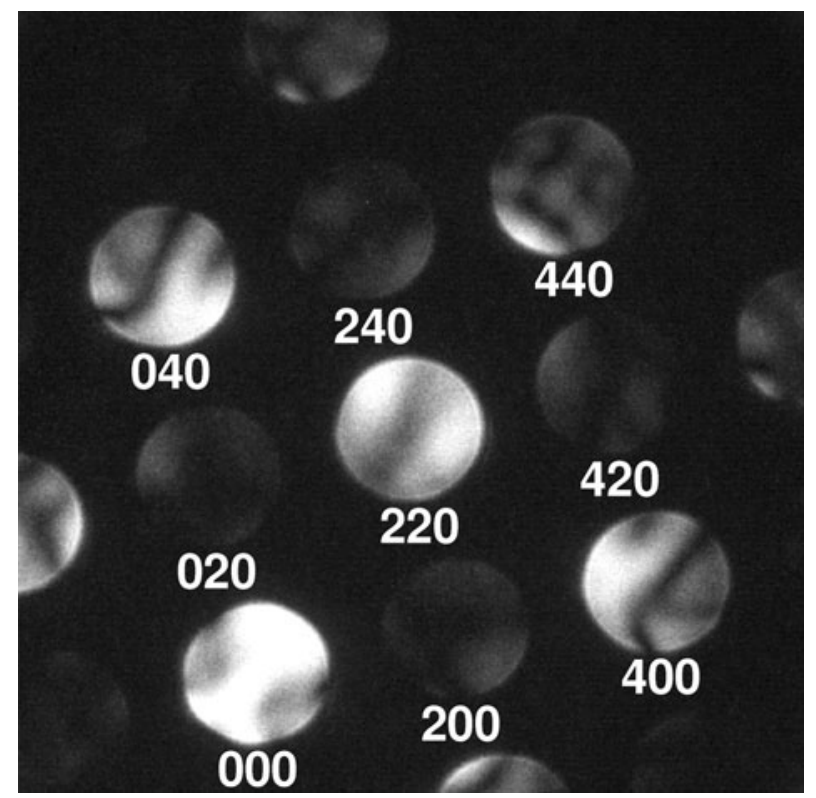

Figure 4. Example for a zero-loss-filtered CBED pattern recorded at $99 \mathrm{~K}$. Due to a small mistilt, energy filtering, and cooling, the reflections appear well-separated from the diffuse background. The size of the pattern is $512 \times 512$ pixels.

the recorded reflections was enhanced by tilting the specimen slightly away from exact [001] zone axis orientation. For the CBED pattern shown in Figure 4, the tilt corresponds to a Laue circle center at (1.5 1.5 0) or, equivalently, an angle of approximately $0.4^{\circ}$. Second, background intensity stemming from thermal diffuse scattering was suppressed by cooling the specimen to $99 \mathrm{~K}$ using a Gatan double tilt cold stage holder (Model 636) filled with liquid nitrogen. Third, electrons having excited plasmons were filtered out using a post-column Gatan Imaging Filter (Tridiem 863) operated in zero-loss mode with a slit width of $10 \mathrm{eV}$. One further series of CBED patterns has been acquired without energy filtering at $300 \mathrm{~K}$. Series sizes have been chosen such that the spatial resolution is limited rather by the probe size than by the sampling of the scanned path, resulting in sampling distances between 0.5 and $0.75 \mathrm{~nm}$ according to Figure 3. Consequently, one dataset includes 600-800 CBED patterns.

\section{Results}

From Figure 4 it becomes obvious that algorithms for (automated) CBED disc position measurement must be robust against the inner disc structure stemming from dynamical scattering. However, common to all discs is that intensity variations due to multiple scattering are restricted to a circular area, leading to an abrupt annular drop of intensity to the background level of the diffraction pattern. Accordingly, algorithms being sensitive to intensity gradients only at the CBED disc boundary can be designed by comprising the circularity of reflections. Therefore, the following three approaches fall into the category of pattern recognition schemes.
The final result of all three methods are CBED disc positions from which local strain in growth direction $\varepsilon_{[100]}$, measured with respect to the GaAs substrate, can be extracted as follows. As mentioned in conjunction with equation (1), the distance vector $\vec{R}_{400}$ between the centers of the 400 reflection and the primary beam is measured in units of pixels. In particular, the location of the latter must be known for each diffraction pattern of a series because it shifts slighty with the position of the incident probe. As this shift is very smooth, we fit a polynomial to the $x$ - and $y$-coordinate of the 000 beam. Expressing $\vec{R}_{400}$ in terms of shifts $\vec{\Delta}_{400}$ from the 400 disc position in unstrained GaAs substrate regions, $\vec{R}_{400}=\vec{R}_{400}^{0}+\vec{\Delta}_{400}$. As strain only occurs along the [100] growth direction in the present case, the vectors can be replaced by their absolute values, which leads to

$$
\varepsilon_{[100]}=\frac{R_{400}^{0}}{R_{400}^{0}+\Delta_{400}}-1
$$

as a simple expression for the measured strain. Note that the determination of all vectors $\vec{R}_{h k l}$ in general provides access to the full bidimensional distortion tensor (Béché et al., 2009). Because our sample exhibits only strain in growth direction, we restrict ourselves on the evaluation of the 400 disc here without loss of generality.

Common to the present methods is the separate treatment of CBED discs. To this end, quadratic patches around each disc are defined manually in one of the diffraction patterns of a series, where also centers and radii of the discs are estimated once by eye.

\section{Selective Edge Detection and Circle Fitting}

\section{The Method}

The above arguments motivate the application of a standard edge detection method to identify pixels on the disc boundary. Here we used the Prewitt approximation (Prewitt, 1970) to the derivative of the intensity distribution in a reflection patch as shown on the left in Figure 5a to obtain edges depicted in the center of Figure 5a. As expected, many edge points accumulate at the disc boundary. On the other hand, the inner disc structure causes edges throughout the whole disc. In the selective edge detection method, the latter are ruled out iteratively until only those edge points remain, which lie on the circumcircle of the CBED disc.

To this end, we first deselect all edge points automatically whose distance to the estimated disc center falls below a certain threshold. This is not a crucial step, but it can speed up the convergence of the algorithm drastically. Whereas usual thresholds are about $80-90 \%$ of the estimated disc radius, we used a value of 50\% here to demonstrate the robustness of the procedure against parasitic edge points in Figure 5b, where squares and circles represent the respective subset of original edge points from Figure 5a. Now position and radius of a circle are fitted to this subset, resulting in the dashed line centered around the open triangle in Figure 5b. The underlying algorithm of Coope (1993) is highly efficient because only a linear least-squares 

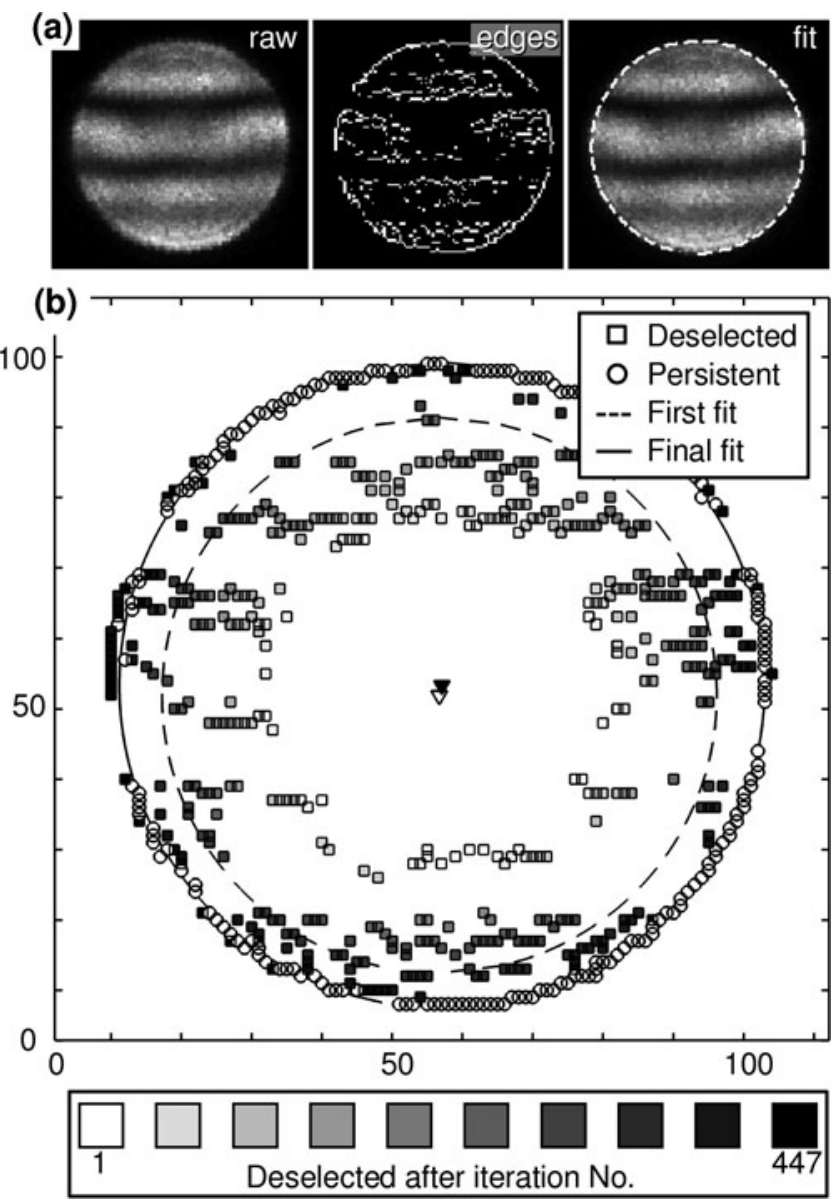

Figure 5. Example evaluation of the 400 CBED disc with the selective edge detection method. a: Shows the original disc, detected edges, and the original disc together with the final edge fit (dashed white line). b: Illustrates how this is achieved: edge points describing the CBED disc's circumcircle are found iteratively. In between the first (dashed) and final (solid line) circle fit, square edge points have been deselected subsequently, the grayscale indicating the respective iteration number. Axes units are in pixels. Centers of the fitted circles are drawn as an open (first fit) and filled (final fit) triangle.

optimization must be performed, but it is also much less sensitive to outliers than a nonlinear least-squares approach. The latter aspect is very important because of the large number of erroneous edge points in the present dataset. Next, the distances of all edge points to this circle are calculated, and the point with the largest distance is deselected for the following fit. This procedure is then iterated until all of the persistent points are as close as a given threshold distance (being one pixel here) to the fitted circle. The iteration in which a certain edge point was deselected from the circle fit is expressed by the gray level of each square in Figure 5b, meaning that dark edge points remained longer. However, a set of points shown as open circles in Figure 5b persists and is subject to the final circle fit shown as a solid line being centered around the filled triangle. Note that the center and the radius of the circle are obtained with subpixel accuracy.

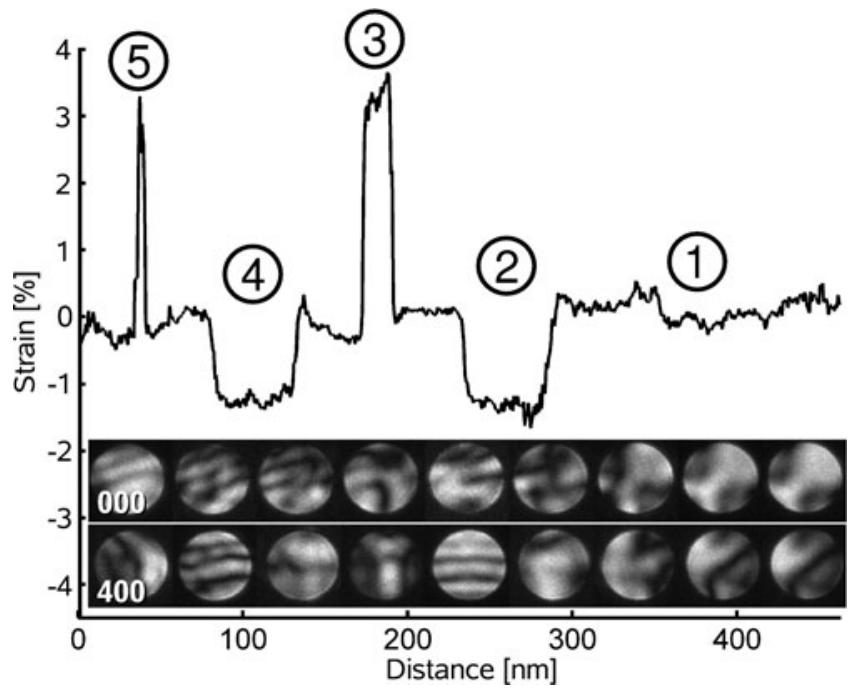

Figure 6. Strain profile along [100] measured by the selective edge detection and circle fitting method. The numbering of the layers corresponds to Figure 1. In the lower part, a selection of 000 and 400 discs is shown to demonstrate that the disc interior varies drastically and individually for each reflection.

Since the radius must be common to all discs of a series, it can be determined from fits to only a few (typically 10-20) discs in a first run. In a second run, it can be kept fixed to the average over the radii of these discs, which speeds up the computation because only circle positions are to be fitted by the algorithm explained above. This is justified because the standard deviation of all radii of the 400 reflection is only 0.5 pixels, being half of the distance that a persistent edge point may have from the final fitted circle. Moreover, keeping the radius fixed improves precision slightly in case only a small fraction of the disc boundary was found by the edge detection preocedure.

To check the reliability of this method by visual control, the result of the selective edge detection and circle fitting method is plotted together with the CBED disc on the right of Figure 5a from which we can draw two important conclusions. First, the fit result is visually pleasing as to an intuitively guessed disc boundary, which secondly verifies the use of a circular pattern afterward. This means that elliptical distortions of the disc due to aberrations of the projection lenses are negligible in the present case, a point that will be addressed quantitatively below.

\section{Results}

Finally, the strain profile was calculated by means of equation (2) and plotted in Figure 6. The quaternary $\mathrm{In}_{x} \mathrm{Ga}_{1-x} \mathrm{~N}_{y} \mathrm{As}_{1-y}$ layer 1 is nearly lattice-matched with small compressive strain of $0.3 \pm 0.1 \%$ in the average. Thus, this measurement exhibits that there are regions in layer 1 where In and $\mathrm{N}$ content are not balanced to a ratio of $8 / 3$, which might be due to the statistical nature of the crystal growth process and which is most likely the reason for the strain gradient directly bottom left of the marker for layer 1. This is followed by the $50 \mathrm{~nm}$ thick ternary $\mathrm{GaN}_{y} \mathrm{As}_{1-y}$ 
Table 1. Composition of Average and Maximum Strain Results for the Strain Profile in Figure 6 (Selective Edge Detection Method). ${ }^{\star}$

\begin{tabular}{lccr}
\hline Layer No. & $\bar{\varepsilon}_{[100]}$ & $\sigma$ & $\varepsilon_{[100]}^{\max }$ \\
\hline 1 & $0.3 \%$ & $0.1 \%$ & $0.53 \%$ \\
2 & $-1.33 \%$ & $0.1 \%$ & $-1.65 \%$ \\
3 & $3.29 \%$ & $0.2 \%$ & $3.64 \%$ \\
4 & $-1.23 \%$ & $0.1 \%$ & $-1.38 \%$ \\
5 & $2.8 \%$ & $0.34 \%$ & $3.29 \%$ \\
\hline
\end{tabular}

${ }^{*}$ The standard deviations $\sigma$ for the average regions are also given.

layer 2 under average tensile strain of $-1.33 \pm 0.1 \%$, being very similar to layer 4 with $\bar{\varepsilon}_{[100]} \approx-1.23 \pm 0.1 \%$. In between we find the $19 \mathrm{~nm}$ thick ternary $\mathrm{In}_{x} \mathrm{Ga}_{1-x}$ As layer 3 compressively strained by $3.29 \pm 0.2 \%$. The last grown quaternary $\mathrm{In}_{x} \mathrm{Ga}_{1-x} \mathrm{~N}_{y} \mathrm{As}_{1-y}$ layer 5 has a thickness of $6 \mathrm{~nm}$ and also exhibits compressive strain, being $2.8 \pm 0.34 \%$ here. Average together with maximum strain values are summarized again in Table 1 for all five layers. In particular, averages and standard deviations have been determined from regions that are certainly inside the layers and do not contain the strain gradients at the onsets and ends. In addition, we determined the precision of this technique from the standard deviation from 50 measurements in the GaAs buffer between layers 1 and 2 to be $7.3 \cdot 10^{-4}$.

Let us at this stage anticipate the most important conclusions concerning this method. The insets in Figure 6 depict a selection of the 000 and the 400 discs of the series, showing not only that specimen thickness and orientation change drastically over a distance of approximately $500 \mathrm{~nm}$, but also that intensity distributions in both discs behave entirely different. Nevertheless, the strain profile exhibits more or less constant precision throughout the whole curve, meaning that the algorithm used here is extremely robust against dynamical scattering affecting the interior of discs. In particular, this is noteworthy because the selective edge detection method requires no outer parameters, except for the initial guess of radius and position of each reflection. Moreover, no filters or changes of the intensity scale are needed. For these reasons, and because the idea of detecting circular edges to characterize a CBED disc position is regarded as a highly intuitive approach, we use the strain profile from Figure 6 as a standard to which the following methods are compared. The reliability of subsequent methods is measured by comparing the positions of the 000 and the 400 discs directly. To this end, a deviation parameter

$$
\delta=\frac{1}{N} \sum_{n=1}^{N} \sqrt{\left(x_{n}^{\text {ref }}-x_{n}\right)^{2}+\left(y_{n}^{\text {ref }}-y_{n}\right)^{2}}
$$

is defined that basically calculates the mean distance between the disc positions $\left(x_{n}^{r e f}, y_{n}^{\text {ref }}\right)$ found in this section and positions $\left(x_{n}, y_{n}\right)$ found by the method under consideration in units of pixel. In equation (3), $n$ indexes one diffraction pattern of a series with length $N$, being 800 here. Though the present method is considered as a reliable standard, it significantly suffers from the partly large num-

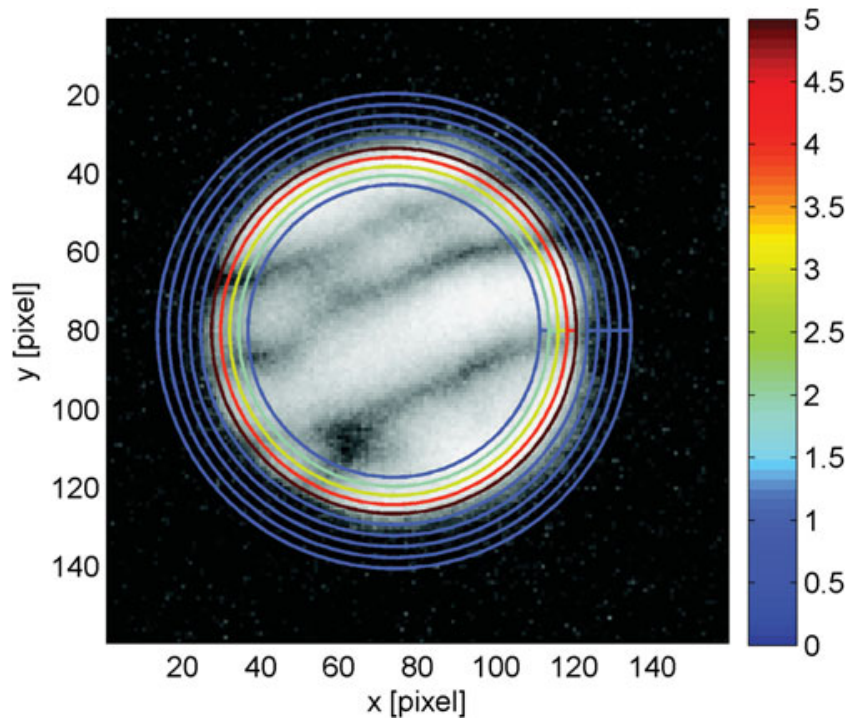

Figure 7. Illustration of the radial gradient maximization method. Image intensity is averaged in regions indicated by the circles inside and outside of the CBED disc. For a better visibility, only 10 circles are shown whereas 40 circles are used in reality. The color code of the circles gives the weight applied to the different circles as described in the text.

ber of iterations necessary to deselect erroneous edge points, being nearly 500 in Figure 5. For example, calculation of the strain profile in Figure 6 took approximately $15 \mathrm{~min}$ on a single $2.88 \mathrm{GHz} \mathrm{CPU}$, whereas the following radial gradient maximization method needed $1 \mathrm{~min}$ and the crosscorrelation methods $12 \mathrm{~s}$.

\section{Radial Gradient Maximization}

\section{The Method}

At least fractions of the CBED disc boundaries exhibit a drastic drop of intensity, which separates disc interior and background as mentioned in the beginning of this section. In contrast to the intensity distribution inside the discs, this step will also occur sharply in rotational intensity averages, provided that the center of this average falls together with the center of the CBED disc. In case the wrong center is used, the rotational average will show up as a blurred step at the disc boundary. From this it follows that the correct disc position can be found by maximizing the intensity gradient in the radial direction, which is the basic idea behind the present method. As illustrated in Figure 7, we introduce two sets of each 20 concentric circles, one set for the region within the CBED disc and one for the exterior region. The algorithm starts by positioning the circles around the estimated disc center and by distributing the 40 radii equally in an interval of $80-130 \%$ of the estimated radius. Then, the mean intensity of all pixels touching the circles is calculated for each circle, which practically represents a rotational average. Finally, we calculate two sums from this rotational average, one from the interior and one from the exterior circles. From the arguments above, it becomes clear that the difference between both sums must be maximized itera- 

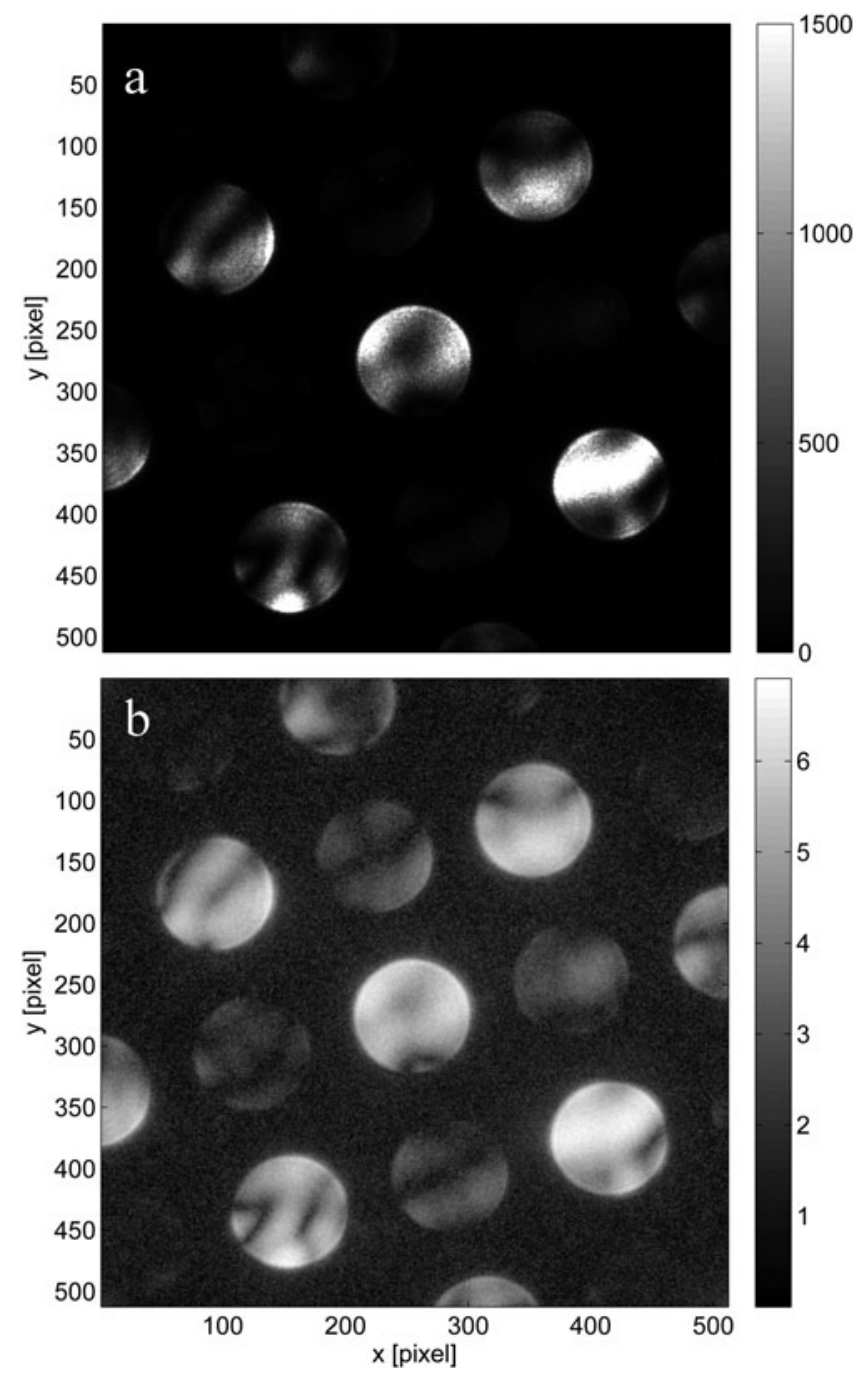

Figure 8. (a) Diffraction pattern from the center of the GaAs region between layers 1 and 2 in comparison with (b) the intensity distribution in the log-scaled image calculated according to equation (4).

tively to find the correct position and radius of a disc. To emphasize the difference between the intensity levels of the disc and the background, we use a logarithmic intensity scaling according to

$$
I_{\log }=\ln (1+I \cdot V)
$$

where $I$ is the original intensity normalized to its maximum, i.e., $\max (I)=1$. By the factor $V$, different logarithmic scalings can be achieved. For the present work, $V=1,000$ has been used. In Figure 8 the log-scale diffraction pattern is compared with the original image.

\section{Results}

Strain profiles determined with the radial gradient maximization method are drawn as the lowermost four graphs in Figure 9, whereas the uppermost curve repeats the result obtained by selective edge detection and circle fitting for comparison. The red curve was obtained by fitting both radius and position of the discs; the green one results from fitting only disc positions and keeping the radii fixed to a value found from fits to the first 10 discs of the series. In case of the blue curve, intensities on the interior circles have additionally been weighted differently so as to stress the impact of circles near the disc boundary. In particular, the weights increased linearly from 1 to 5 toward the largest interior circle as expressed by the color coding in Figure 7. Note that no weighting scheme is applied for the exterior circles. As announced in the previous section, we also checked whether elliptical distortions of CBED discs need to be taken into account by replacing the circles with ellipses in which the orientation and ellipticity have been determined from fits to the first 10 discs of our dataset. In the present case we found the values 45.8 and 47.1 pixels for the shortest and longest axis of the ellipse. The evaluation result is expressed by the light blue, lowermost graph in Figure 9.

On the whole, all graphs in Figure 9 represent the character of the layer stack with alternating compressive and tensile stress correctly, albeit with different precisions and a few outliers in part. In this respect, the red and the green curves exhibit artifacts in layers 2 and 4 , where tensile strain is under- or overestimated near the well centers, respectively. In contrast, the blue and light blue curves look very similar to each other and to the black standard. As we are aiming at the minimization of $\delta$ in equation (3), this parameter is additionally given in the legend of Figure 9. From this we conclude that keeping the circle radii fixed to a value found from the first discs improves the agreement between the reference and the present method slighty from $\delta=0.67$ pixel to $\delta=0.65$ pixel. Contrarily, linearly increasing the weights of interior circles radially has much more impact because $\delta$ drops to a value of 0.52 pixel. A visual comparison of the blue and green graphs exhibits that this decrease of $\delta$ by more than 0.1 is mainly due to the missing outliers inside the GaNAs layers. Consequently, this weighting scheme enhances the radial gradient maximization method significantly. It is interesting to see that fitting ellipses leads to an increase to $\delta=0.58$ pixel again, meaning that adding two more fit parameters (orientation and ellipticity) results in less accurate disc positions. However, the precisions of both methods calculated in the same region as in the case of selective edge detection and circle fitting yield almost the same value of $8.5 \cdot 10^{-4}$.

\section{Cross Correlation with Masks}

\section{The Method}

Instead of detecting the absolute disc positions inside a patch around a CBED reflection, we can also think of measuring only relative shifts with respect to an arbitrary origin inside the patch. The consequence of this seemingly marginal difference to the preceding two sections is that we can use the highly efficient formalism of cross correlation if we place an object with sufficient similarity to the CBED disc under consideration at this origin. It is clear that similarity refers to the position of the disc boundary in this 


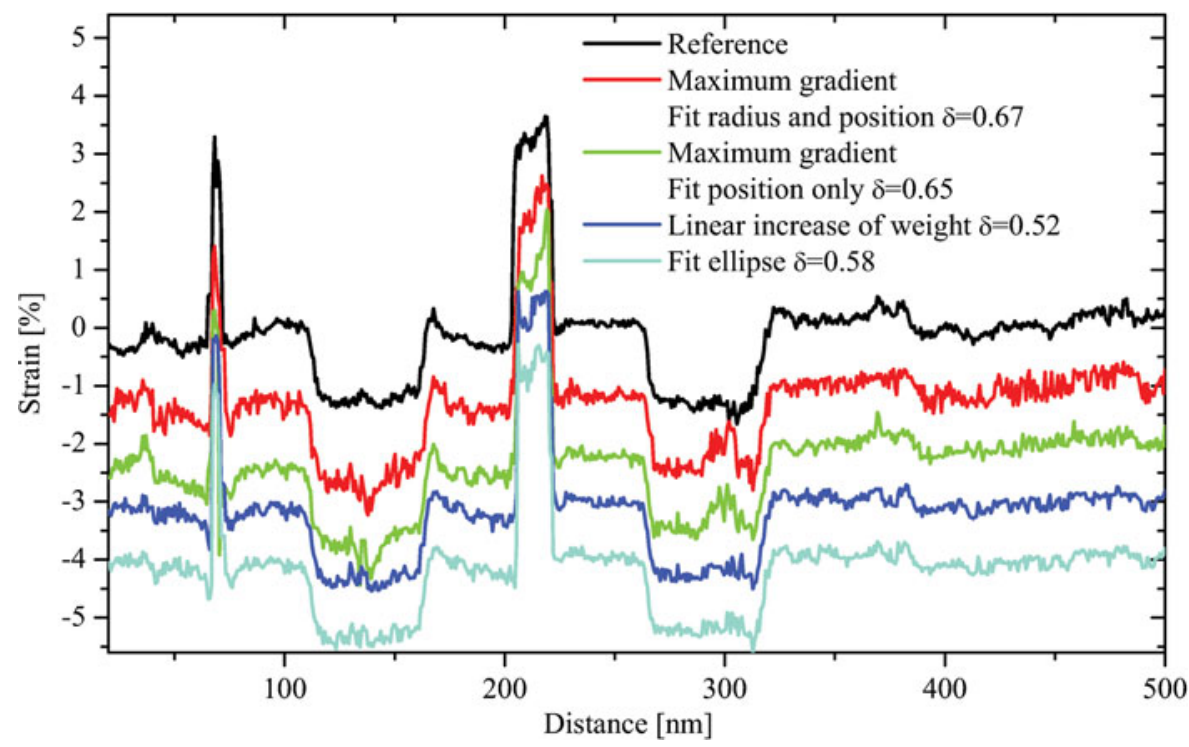

Figure 9. Strain profiles along [100] measured by radial gradient maximization. The individual graphs are shifted with respect to each other to improve their visibility. The uppermost (black) graph shows the reference curve obtained by selective edge detection and circle fitting. Details of the profiles below are given in the legend. Deviations $\delta$ from the selective edge detection method are also given according to equation (3) in units of pixel. (In gray-level representation, the color ordering is, from top to bottom: black, red, green, blue, light blue.)

work, so that we report on results obtained by crosscorrelation of the respective patch with patches containing several types of masks designed to diminish the impact of the intensity distribution inside the discs.

As for the radial gradient maximization method, we use logarithmic intensity scaling according to equation (4) and determine the radius of all masks shown in Figure 10 by radial gradient maximization applied to the diffraction patterns $1-10$ of the series. To achieve subpixel accuracy, a $10 \times 10$ pixels $^{2}$ large patch is cut out of the crosscorrelation image around the pixel with highest intensity. Then the pixel values within the patch are summed up along the horizontal direction, and the resulting intensity line scan is fitted with a fifth-order polynomial. Finally, the global maximum of the polynomial is used as $x$ coordinate of the maximum position. The same is repeated for the vertical direction, which gives the $y$-coordinate with subpixel accuracy. Before discussing the results explicitly, we first motivate the type of masks displayed in Figure 10. The use of a simple aperture function being one inside and zero outside a circular area (Fig. 10a) equal to the CBED disc size goes back to the scattering properties of a specimen with very low mass thickness as its diffraction pattern will mainly consist of more or less homogeneously illuminated discs. As expressed by Figures 4 and 6, this is a too crude approximation here. Disregarding the innermost part completely should therefore enhance sensitivity on the disc position, which is why we used the ring-shaped mask as a second object (Fig. 10b). Moreover, it was found for the radial gradient maximization method that linearly increasing the weight radially yields the best results, which motivated the use of the third mask shown in Figure 10c.
Results

Figure 11 shows the strain profiles obtained by cross correlation with masks shown in Figure 10, together with a study of the dependence of the inner radius in case of the annular mask with radially increasing weight. Again, the result of selective edge detection and circle fitting is repeated to facilitate a comparison (black graph). As expected, cross correlation with a filled disc (red graph) gives erroneous results as can be seen from the global slope the curve shows, the peaks and dips in layers 2 and 4, respectively, as well as from the wrong representation of well 5 on the left. Consequently, the deviation of $\delta=1.3$ pixels determined according to equation (3) is more than twice as large as for the other curves. They in turn look very similar to each other, except for a slight underestimation of tensile strain in layer 2 when an annular mask without weighting scheme is used. However, small differences become obvious by means of the deviations $\delta$, taking a minimum of 0.56 pixels for the weighted annular mask whose inner radius $r_{i}$ is $80 \%$ of the CBED disc. To check whether the choice of this value is critical, evaluations have been repeated with $r_{i}=0.9$ and 0.7 , which raised the parameter $\delta$ slightly. As in the preceeding sections, we calculated the precision of the crosscorrelation method to be $7.8 \cdot 10^{-4}$ from a region between layers 1 and 2 when an annular mask with $r_{i}=0.8$ and linearly increasing weight in radial direction is used.

\section{Cross Correlation with Central Disc}

\section{Original Method}

Figure 12 depicts the results of evaluations carried out according to the method suggested by Uesugi et al. (2011). As described in this article, high-pass filtering of the origi- 

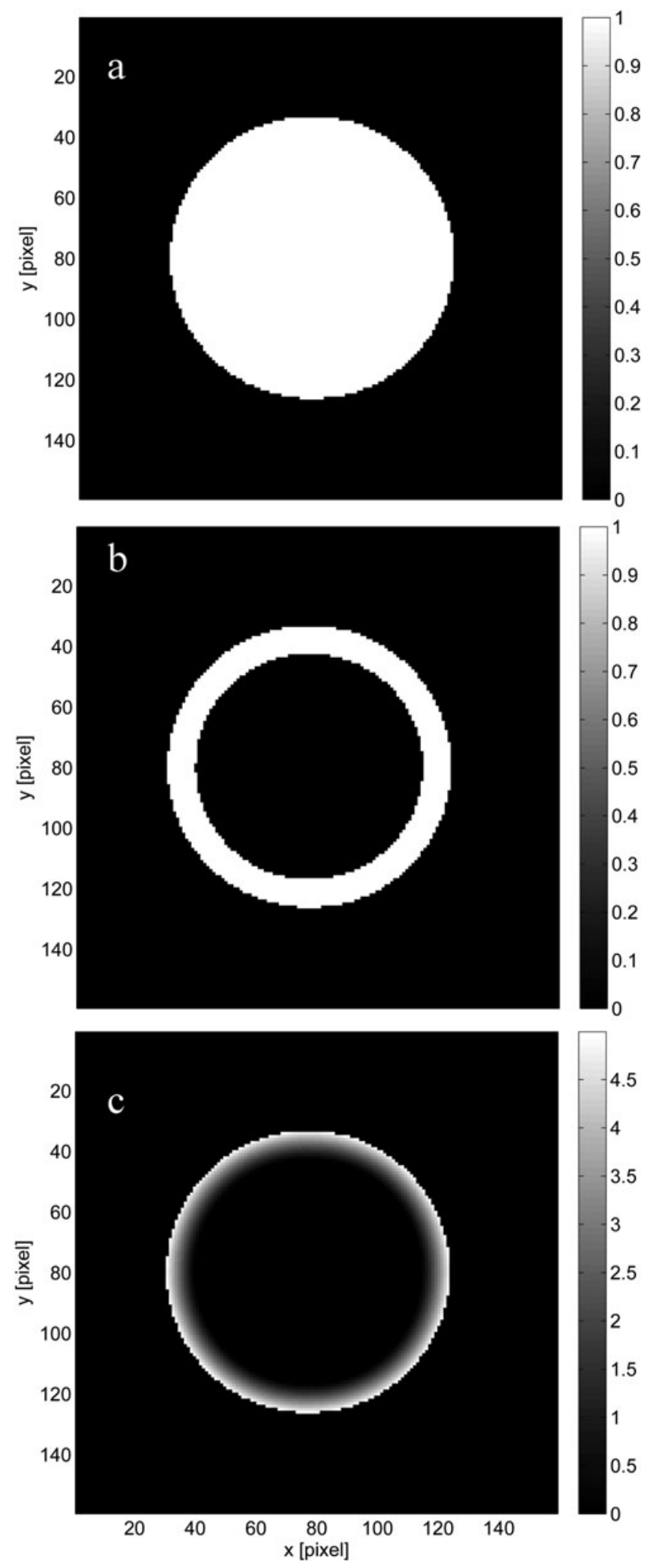

Figure 10. Masks used for cross correlation: (a) disc-shaped mask, (b) ring-shaped mask, and (c) weighted ring-shaped mask.

nal diffraction patterns (without logarithmic intensity scaling) was performed by setting all pixels within a discshaped area around the centers of the Fourier-transformed images to zero. The sizes of the discs used are given in the legend of Figure 12. Then, two patches of the same size and position were cut out of each diffraction pattern, one of which was centered around the undiffracted beam and the other one was centered around the 400 beam. Finally, cross correlations of these patches were computed for all diffraction patterns, and the shifts between undiffracted beam and 400 beam were obtained with subpixel accuracy.

\section{Results}

To investigate the accuracy of the method, we tested highpass filtering using discs with sizes between 20 and 50 pixels. Too weak high-pass filtering leads to a strong scattering of the evaluated strain as can be seen from the green graph corresponding to a disc radius of 20 pixels. This scattering of data points is connected with a large value of the deviation parameter of $\delta=18.2$ pixel. Large deviation parameters are also found for disc radii larger than 40 pixels, due to loss of information in the high-pass filtered images. The best result was reached with a disc radius of 30 pixels, leading to a deviation parameter of $\delta=3$ pixels. To give an impression of the strength of the high-pass filtering needed, the high-pass filtered image obtained with a disc radius of 30 pixels is shown in Figure 13a. In the case of the 30 pixel disc, we computed the precision of the method as in the preceeding sections between layer 1 and 2 and found a value of $6 \cdot 10^{-4}$. Although this precision calculated in a GaAs region is good, incorrect strain values are found in all layers 2 to 5 . An advantage of this method is that it is not necessary to take the shift of the undiffracted beam into account separately as described above as it evaluates relative shifts between the undiffracted and diffracted beams.

\section{Logarithmic Intensity Scaling}

Finally, we checked whether logarithmic scaling of images prior to high-pass filtering would improve the stability of the strain evaluation. As in the case of the original method, we varied the radii of the disc-shaped masks used for high-pass filtering, here between 12 and 50 pixels as depicted in Figure 14. In comparison to Figure 12, the strain profiles appear more noisy, which can be expected due to the increase of noise induced by log scaling the diffraction patterns combined with high-pass Fourier filtering. However, in the regions of layers 2 to 5 , outliers disappear so that we conclude that the method becomes more stable. Again, we find best agreement with the reference graph (black) for the 30 pixel disc mask. The corresponding high-pass filtered diffraction pattern is shown in Figure 13. The precision measured between layers 1 and 2 is $1.6 \cdot 10^{-3}$.

\section{Influence of Energy Filtering and Specimen Cooling}

All results shown so far have been evaluated from the same series of diffraction patterns recorded under optimum conditions, i.e., cooling the sample to liquid nitrogen temperature and using an energy filter that selects electrons with an energy loss smaller than $10 \mathrm{eV}$. Low sample temperature decreases the background signal in a diffraction pattern and increases the intensity of Bragg reflections due to small 


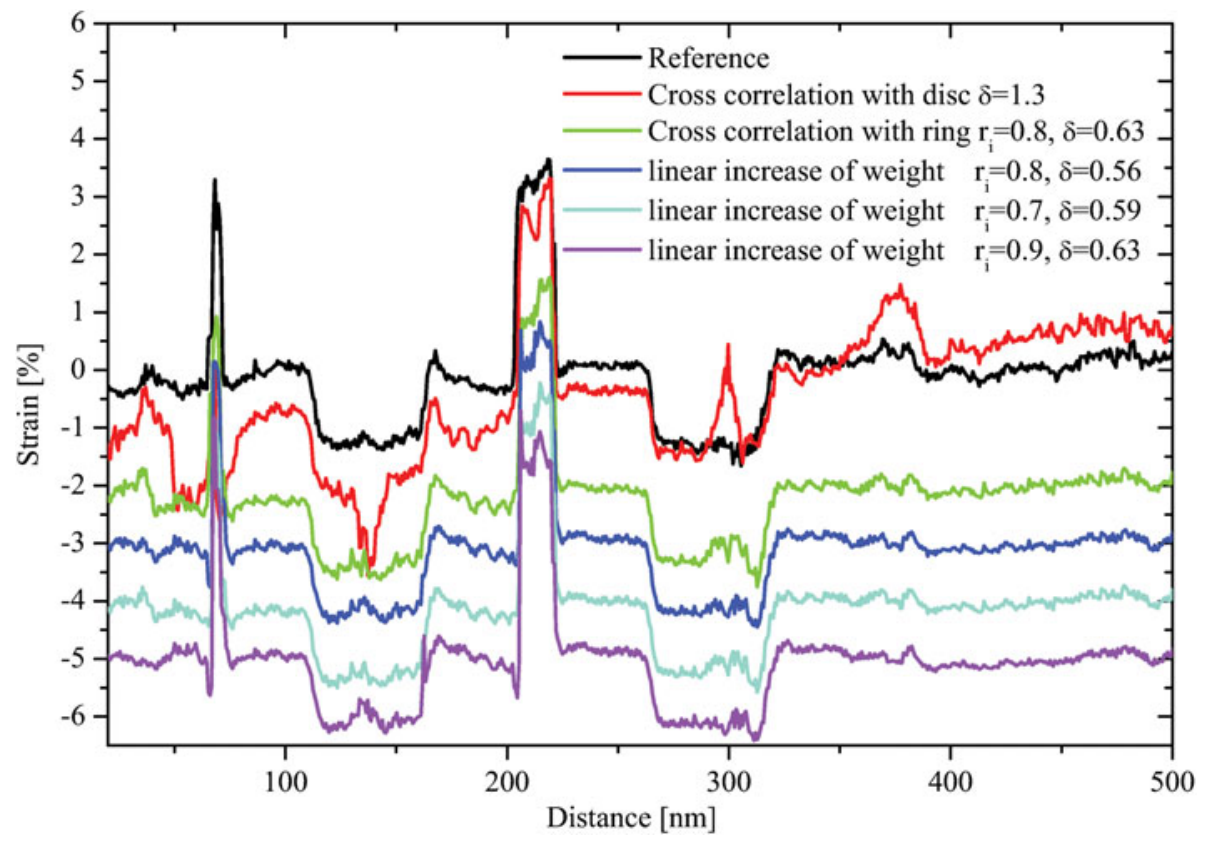

Figure 11. Strain profiles along [100] measured by cross correlation with masks of different type. The red curve corresponds to the disc-shaped mask (Fig. 10a), the green curve to the ring-shaped mask (Fig. 10b), and the following curves were evaluated with the weighted ring-shaped mask (Fig. 10c) for different radii $r_{i}$ of the inner hole of the mask, given in units of the radius of the CBED disc. (In gray-level representation, the color ordering is, from top to bottom: black, red, green, blue, light blue, magenta.)

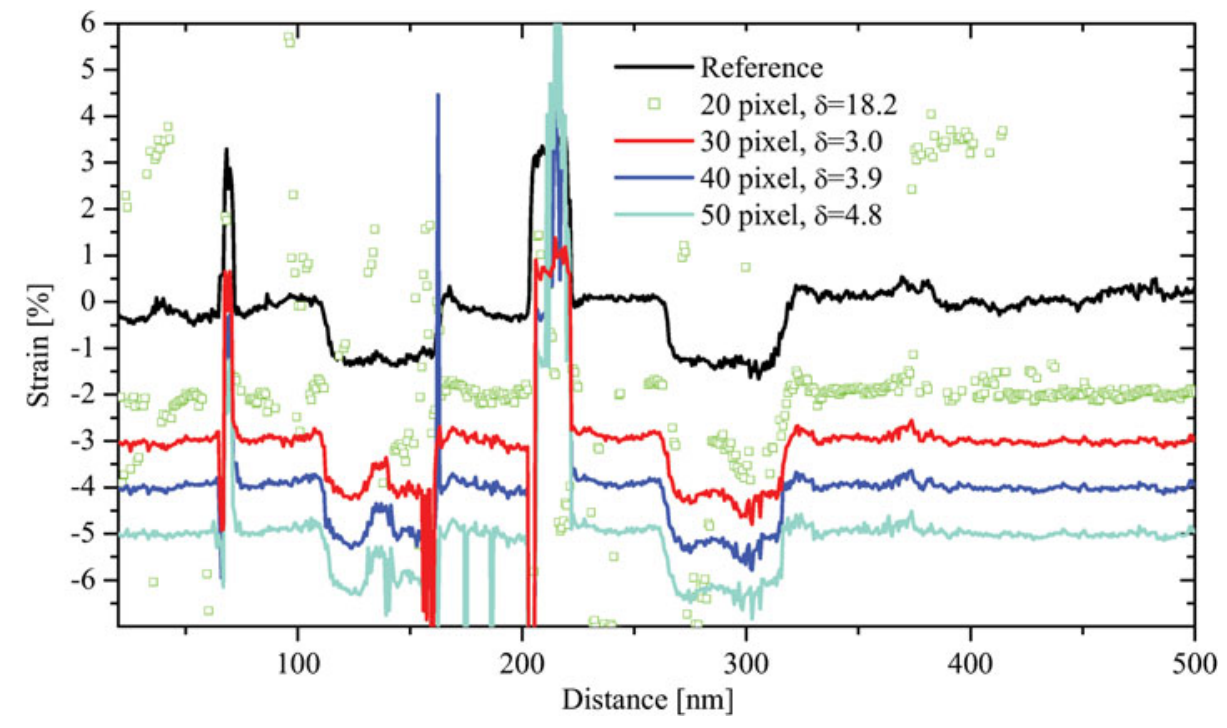

Figure 12. Strain profiles along [100] measured by cross correlation between the 400 and the central disc in the original diffraction pattern. The values in pixels indicate the strengths of the high-pass filtering by giving the radii of the discs removed from the Fourier-transformed diffraction patterns, which were centered around the origin. For the green graph, squares are drawn instead of a line graph as the high spread of values would reduce the visibility of all the other graphs. (In gray-level representation, the color ordering is, from top to bottom: black, green, red, blue, light blue.)

mean thermal displacements and thus small Debye-Waller temperature factors. The energy filter especially removes electrons having excited plasmons from the imaging process. These electrons lead to a blurring of the diffraction pattern as plasmon excitation is connected with small but disturbing momentum transfer. In this section we investigate the accuracy of strain evaluation with the methods presented in the preceding sections under nonoptimum conditions, where the same sample as used before was kept at room temperature and the electron energy filter was not used. Figure 15 shows the diffraction pattern mainly revealing the 000 and 400 reflection; the 200 reflection is hardly visible. The series contains 600 diffraction patterns and was evaluated with different methods. The results are shown in 


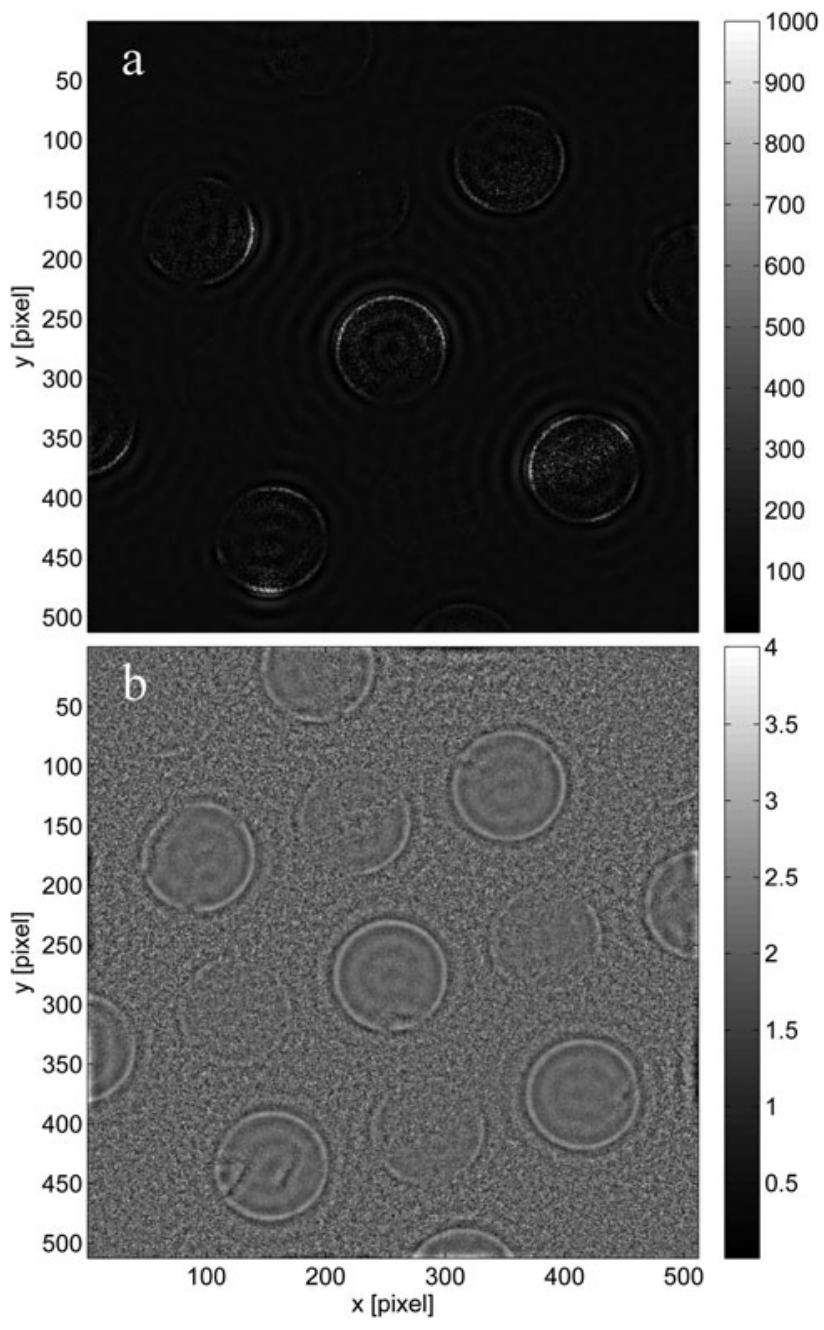

Figure 13. Diffraction pattern of the series after high-pass filtering using a disc-shaped area of 30 pixel diameter in which the Fourier components of the Fourier-transformed image were set to zero. (a) Corresponds to the high-pass filtered image of the original diffraction pattern and (b) is the high-pass filtered image of the diffraction pattern after logarithmic intensity scaling.

Figure 16. The black curve obtained with the selective edge detection and circle fitting method is used as the reference graph as before. Although the diffraction patterns were taken at nonoptimum conditions, the quality of the resulting strain profile is similar to the profile obtained under optimum conditions. The main difference is the occurrence of fluctuations in layer 4, which could be caused by random alloy fluctuations. The strain profiles evaluated with the maximum gradient (red) and cross-correlation method using a weighted ring-shaped mask (green) are in a good agreement with the reference profile. The cross correlation with the central disc (blue dots) leads to large deviations especially in layer 3. This artifact does not show up if the log-scale diffraction pattern is used. In conclusion, cooling of the specimen and application of energy filtering are not necessary for the investigated methods as similar results are obtained if the specimen is kept under room temperature and energy filtering is omitted.

\section{DIsCUSSION}

In the preceding section three different methods have been developed, which lead to nearly identical strain results if governing parameters for the radial gradient maximization and the cross correlation with masks are chosen properly. On the one hand, using the edge detection method as a standard to which the other results are compared appears plausible because it works parameter-free and is applied to raw data. For radial gradient maximization and cross correlations, the situation is different: First, we observe different strain results depending on whether we use, for example, a radial weighting scheme or not. Second, for the cross correlations different types of masks are used, with or without weighting scheme, and the inner ring radius as an additional parameter enters in which the actual value has to be verified, or at least it has to be shown that its choice is not critical. Third, cross-correlation methods compare the similarity of two objects. Inventing masks as shown in Figure 10 therefore in principal claims that the experimental patches are similar to these masks except for a shift, which is obviously not true. Consequently, choosing the selective edge detection method that does not suffer from these issues was intended to validate cross correlation and radial gradient maximization. On the other hand, care must be taken when using a method as a standard because it could preclude other methods to perform better. In the sense of strain state analysis, this would mean lower statistical fluctuations of the strain curve. This would not necessarily result in a decrease of the parameter $\delta$ but in a lower standard deviation. For this reason, one should look at both $\delta$ and the precision simultaneously to judge whether a method performs better. In our case, all three precisions are comparable, and there is no reason to claim that one of the methods performs best in terms of accuracy and precision. However, in practice a fast algorithm is favorable, so that cross-correlation-based strain state analysis may be preferable. Finally, the three methods have been compared without defining one of them as a standard by application to a simulated GaAs CBED pattern. To account for thermal diffuse scattered electrons in the background, the frozen lattice multislice approach was used with thermal displacements corresponding to $300 \mathrm{~K}$ and a specimen thickness of $100 \mathrm{~nm}$. Disc positions have been evaluated by selective edge detection and circle fitting, radial gradient maximization, and cross correlation with masks as described above. According to equation (2), precisions and accuracies for strain evaluation have been determined for the three approaches. Similar to the present experiment, precisions deviated less than $2.2 \cdot 10^{-4}$ among the three methods. As to accuracies, maximum differences of $4 \cdot 10^{-4}$ have been found between selective edge detection and cross correlation with a weighted ring mask.

Since the strain results of the three methods are equivalent, we discuss only the reference profile in Figure 6 substitutionally for the result of all methods in the following. Main features we see in this profile are well-resolved, 


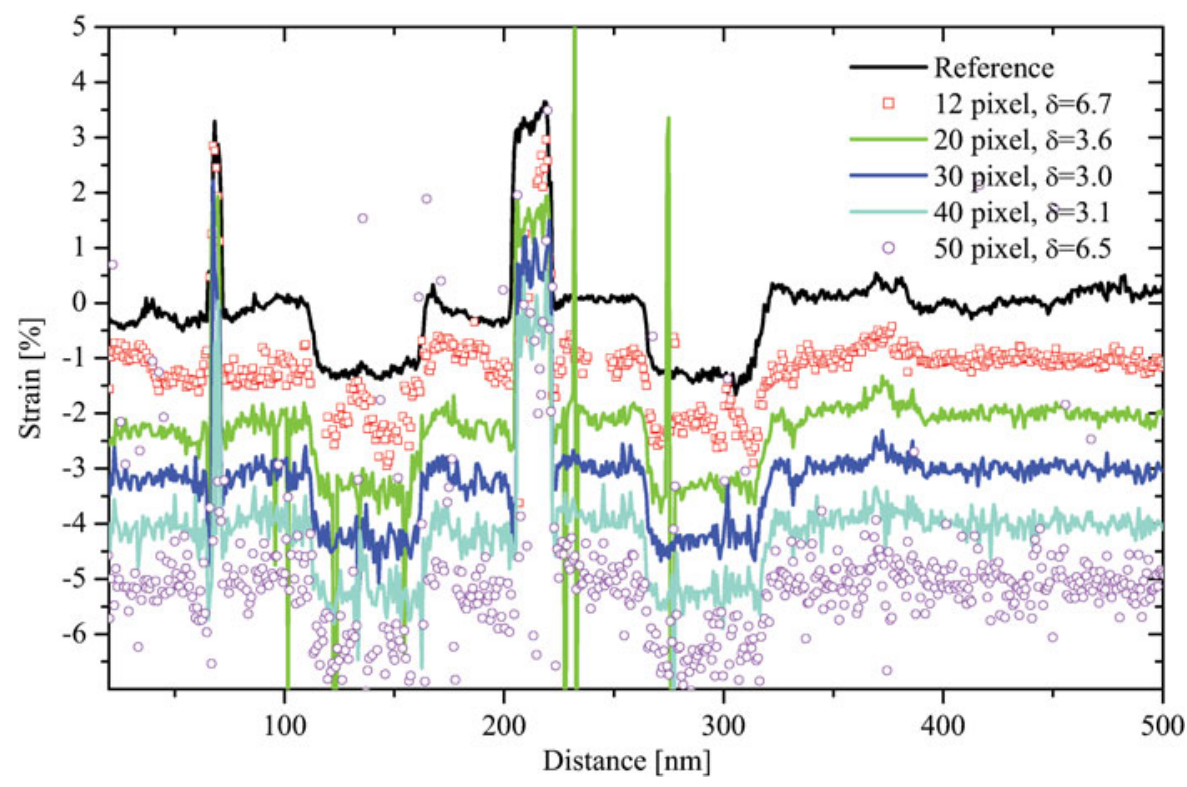

Figure 14. Strain profiles along [100] measured by cross correlation between the 400 and the central disc using logarithmic intensity scaling according to equation (4). The values in pixels indicate the strengths of the high-pass filtering by giving the radii of the discs removed from the Fourier-transformed diffraction patterns, which were centered around the origin. To improve visibility, symbols are used instead of lines for the graphs corresponding to radii of 12 and 50 pixels. (In gray-level representation, the color ordering is, from top to bottom: black, red, green, blue, light blue, magenta.)

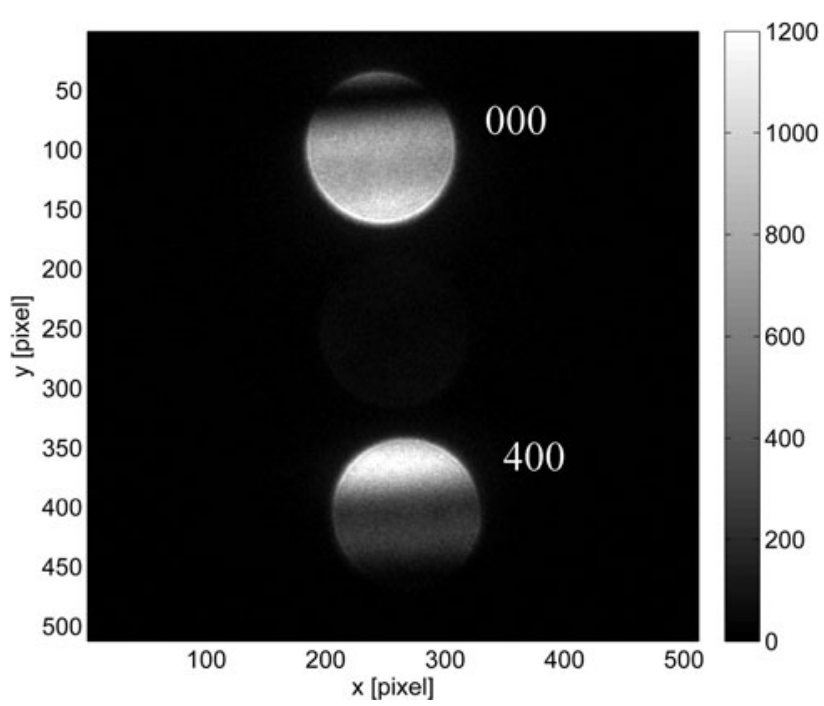

Figure 15. Diffraction pattern obtained without energy filtering or specimen cooling from the center of the GaAs region between layers 1 and 2.

alternating compressively, and tensile strained layers with sharp interfaces. Moreover, the profile as a whole does not exhibit a biasing or slope. The fact that we observe nearly unstrained regions in between the layers furthermore proves that lattice plane bending at the specimen surface influences the measurement only marginally and that thin foil relaxation plays a minor role here, being in agreement with a specimen thickness of $100 \mathrm{~nm}$. One very important issue is that the dimension of the profile covers approximately $500 \mathrm{~nm}$, throughout which orientation and thickness change significantly according to the reflections in Figure 6 without altering the precision of the strain measurement. Except for nanobeam diffraction (NBD) experiments (Usuda et al., 2005; Armigliato et al., 2008; Liu et al., 2008; Béché et al., 2009; Favia et al., 2011) or, more precisely, all techniques that exploit the position of Bragg beams, both classes of methods based either on the measurement of Bragg beam phases or on evaluation of HOLZ line intersections can suffer from strong variations of crystallographic orientation. This is because the former interprets the beam phase solely in terms of strain, and the latter is reliant on the permanent presence of the highly orientation-dependent HOLZ lines under consideration in a certain CBED disc, which is why this technique often applies to specimens being three times thicker than the present one (Favia et al., 2011).

Herewith we arrived at the discussion of parameters that govern the precision of strain measurements. Owing to Bragg's law (1), none but the local lattice parameter determines the measured signal in diffraction-based techniques so that the precision is to be discussed rather by means of the analyzing procedure applied to the diffraction patterns than by inaccurately known specimen parameters, such as its thickness, orientation, composition, and crystal potential Fourier components [which are sensitive to bonding (Rosenauer et al., 2005), thermal (Waller, 1927), and static disorder (Glas, 2004; Müller et al., 2010)] as mentioned in the introduction. Precisions obtained from diffraction-based techniques and those relying on Bragg beam phase evaluations are therefore compared separately. The precision of $7.3 \cdot 10^{-4}$ reported here belongs to the highest ones obtained from reflection position measurement as a recent overview in Favia et al. (2011) gives values between $6 \cdot 10^{-4}$ and $1 \cdot 10^{-3}$ for NBD methods. Similarly, HOLZ line analyses can reach precisions up to $1.5 \cdot 10^{-4}$.

The highest precisions for strain measurements at large fields of view the authors are aware of are based on darkfield holography (Hÿtch et al., 2008; Hüe et al., 2009; Koch et al., 2010). It turned out that it is extremely difficult to compare with these reports for two reasons, one physical and one methodical. Physically, none of the references addresses the impact of specimen and imaging parameters that govern diffracted beam phase apart from strain fields, 


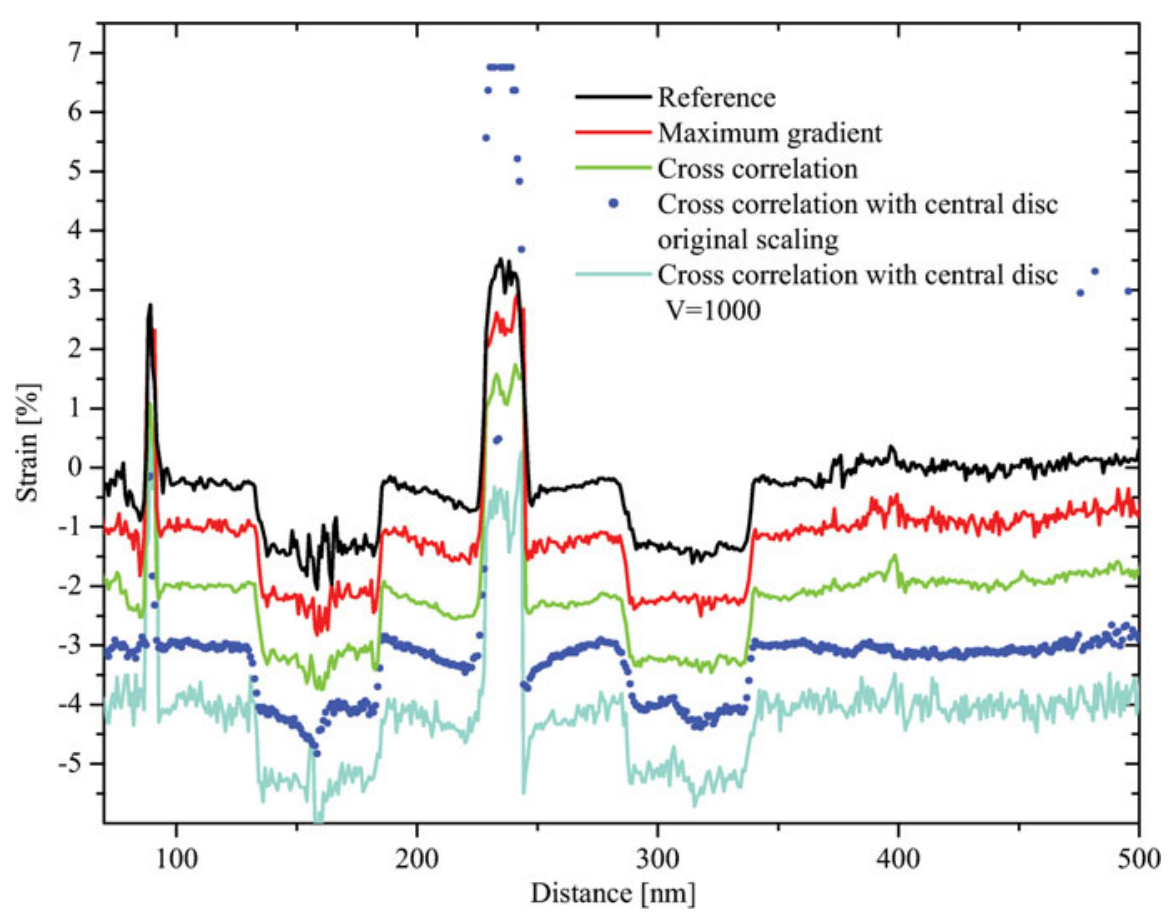

Figure 16. Strain profiles along [100] measured without energy filtering and keeping the sample at room temperature. The evaluations were performed with different methods: selective edge detection and circle fitting method (black), maximum gradient method using an inner circle radius of 0.8 times the CBED disc size (red), cross correlation with a weighted ring-shaped mask (green), cross correlation of the diffracted disc with the central disc using the originally scaled image (blue), and using the log-scaled image (light blue). (In gray-level representation, the color ordering is, from top to bottom: black, red, green, blue, light blue.)

especially orientation-dependent behavior of the phase in presence of thickness and composition gradients, the latter being the reason for chemical shifts frequently observed in high-resolution TEM. In addition, precisions are derived in different ways in literature. However, giving the standard deviation among independent measurements that physically ought to yield identical results is regarded as the most reliable way by the authors, which is why we derived our value from an ensemble of 50 diffraction patterns. As to dark-field holographic techniques, this allows direct comparison only with the precision of $2 \cdot 10^{-3}$ determined from the standard deviation in the substrate region of the strain map (Hÿtch et al., 2008). Hüe et al. (2009) calculated the precision of $2 \cdot 10^{-4}$ by first deriving five strain profiles averaged over $65 \mathrm{~nm}$ wide regions and then giving the standard deviation among these profiles. Our data do not allow for this treatment as we only recorded a one-dimensional profile, but averaging over tens of nanometers before calculating the standard deviation is supposed to increase the precision significantly. Finally, Koch et al. (2010) report on a precision of $1 \cdot 10^{-5}$, whereas the authors lack details on its derivation, which is also why this value might not be compared directly with the present report.

As to the spatial resolution of our technique, we gave a value of $0.5-0.7 \mathrm{~nm}$ obtained from spot profile and exit wave simulations. In comparison to diffraction-based techniques achieving comparable precisions, the spatial resolution in the present work is approximately five times better than in NBD experiments (Béché et al., 2009). However, our analyses were based on the evaluation of the 400 disc because the adjacent reflections 200 and 600 are extremely weak, as expressed by Figure 15. Consequently, the semiconvergence of the incident probe could be raised further to a value slightly below the Bragg angle of the 400 beam, which could allow for the resolution of 200 lattice planes.

Scanning CBED and NBD experiments currently suffer from two things, the large amount of data needed for the postprocessing of the diffraction pattern series and the limited speed of the CCD camera. The first issue can in principle be solved by performing all evaluations (that is, the first three algorithms proposed in the Results section) in situ at the microscope at the CBED pattern just acquired, while the acquisition of the next one is running. Second, detectors faster than the CCD used here are preferable, such as direct electron detectors or detectors working on the delay-line principle as used in particle physics.

Although this is very promising, one can also think of using existing, ultrafast TEM hardware, especially scanning TEM bright-field detectors. In this case, the distinct intensity distribution in the diffraction pattern is lost, so that the algorithms used here must be translated to hardware operations, which is briefly sketched in the following. First, we have seen that energy filtering is not mandatory, meaning that a bright-field detector, typically mounted in front of a Gatan spectrometer, can be used. Now consider a diffracted beam, e.g., the 400 disc in the present study, being exactly as 
large as the detector (which is practicable in a threecondenser lens setup as shown in the Materials and Methods section) centered on this detector (which maximizes the recorded bright-field signal) by the diffraction shift deflection coils. In case this disc is changing position due to a variation of the lattice constant in the specimen, the diffraction shift setting must be changed to get the maximum signal on the bright-field detector. In most TEM, scripting adapters allow for an automated finding of this shift, being proportional to the strain and which can be mapped versus the probe position. Except for the logarithmic intensity scaling, this procedure ought to be analogous to the crosscorrelation method using the mask from Figure 10a. Placing a ring-shaped aperture above the detector is then analogous to using the mask from Figure $10 \mathrm{~b}$ and means a minor modification of the hardware.

A central problem dealt with in the present article was the inner structure of the CBED discs in which the influence has been minimized by weighting schemes or searching for edges on a circle, which is difficult to translate to a hardware setting for the bright-field detector. However, one could alternatively also precess the incident beam around the optical axis of the TEM and compensate for the movement of the diffraction pattern by the diffraction shift setting. By means of Bloch wave simulations, the authors checked that this leads to nearly homogeneously illuminated CBED discs, so that an accurate strain measurement should be possible by recentering a diffracted beam on a bright-field detector in precession mode. Moreover, similar results can be expected when electron precession is combined with a four-quadrant bright-field detector as used for differential phase contrast (Chapman, 1984; Ploessl et al., 1993) measurements to quantify magnetic and electric fields. It is worth noting that strain measurement based on cross correlation of two discs (Uesugi et al., 2011) in the same CBED pattern is difficult to translate to a hardware setup.

\section{Summary AND CONCLUSIONS}

Based on the evaluation of disc positions in CBED patterns acquired serially, strain profiles in a highly compressively and tensile strained $\operatorname{In}_{x} \mathrm{Ga}_{1-x} \mathrm{~N}_{y} \mathrm{As}_{1-y} / \mathrm{GaAs}$ heterostructure consisting of five layers have been measured with a precision of $7-8 \cdot 10^{-4}$ and a spatial resolution of $0.5-0.7 \mathrm{~nm}$. Identical profiles have been obtained by three algorithms aiming at the recognition of a circular pattern centered around a diffracted beam. The most efficient techniques are based on maximizing intensity gradients in rotational averages of a disc or on cross correlation with ring-shaped masks. The choice of governing parameters for these methods has been verified by comparing with a parameter-free method based on edge detection and circle fitting.

In conclusion, the precision of our techniques is comparable to the highest ones achieved by scanning CBED or NBD approaches while increasing the spatial resolution by a factor of 5, which makes them an ideal tool for the investi- gation of contemporary semiconductor nanostructures such as MOSFETs, light emitting or laser diodes of comparable complexity as in the present study. The fact that Bragg beam positions are solely determined by the local lattice constant in field-free crystals allows a direct interpretation of the results in terms of strain, contrary to high-resolution TEM or holographic methods that exploit Bragg beam phases, being dependent on at least five parameters. From the methods designed here, TEM hardware setups as well as microscope control software features have been proposed for future experiments.

\section{ACKNOWLEDGMENTS}

This work was supported by the Deutsche Forschungsgemeinschaft (DFG) under contract numbers RO2057/8-1, SCHO1196/3, V0805/4, and V0805/5.

\section{REFERENCES}

Armigliato, A., Frabboni, S. \& Gazzadi, G.C. (2008). Electron diffraction with ten nanometer beam size for strain analysis of nanodevices. Appl Phys Lett 93(16), 161906.

Bayle, P., Deutsch, T., Gilles, B., Lançon, F., Marty, A. \& Thibault, J. (1994). Quantitative analysis of the deformation and chemical profiles of strained multilayers. Ultramicroscopy 56(1-3), 94-107.

Béché, A., Rouvière, J.L., Clément, L. \& Hartmann, J.M. (2009). Improved precision in strain measurement using nanobeam electron diffraction. Appl Phys Lett 95(12), 123114.

Bierwolf, R., Hohenstein, M., Phillipp, F., Brandt, O., Crook, G. \& Ploog, K. (1993). Direct measurement of local lattice distortions in strained layer structures by HREM. Ultramicroscopy 49(1-4), 273-285.

BragG, W.H. \& BragG, W.L. (1913). The reflection of X-rays by crystals. Proc Roy Soc Lond A 88, 428-438.

Chapman, J.N. (1984). The investigation of magnetic domain structures in thin foils by electron microscopy. J Phys D: Appl Phys 17(4), 623.

Coope, I.D. (1993). Circle fitting by linear and nonlinear least squares. J Opt Theory Appl 76, 381-388.

Favia, P., Gonzales, M.B., Simoen, E., Verheyen, P., Klenov, D. \& Bender, H. (2011). Nanobeam diffraction: Technique evaluation and strain measurement on complementary metal oxide semiconductor devices. J Electrochem Soc 158(4), H438-H446.

Glas, F. (2004). The effect of the static atomic displacements on the structure factors of weak reflections in cubic semiconductor alloys. Philos Mag 84(20), 2055-2074.

Guerrero, E., Galindo, P., Yáñez, A., Ben, T. \& Molina, S.I. (2007). Error quantification in strain mapping methods. Microsc Microanal 13(5), 320-328.

Hüe, F., HŸtch, M., Houdellier, F., Bender, H. \& Claverie, A. (2009). Strain mapping of tensiley strained silicon transistors with embedded $\mathrm{Si}_{1-y} \mathrm{C}_{y}$ source and drain by dark-field holography. Appl Phys Lett 95(7), 073103.

Hั̈тch, M., Houdellier, F., Hüe, F. \& Snoeck, E. (2008). Nanoscale holographic interferometry for strain measurements in electronic devices. Nature 453, 1086-1089.

Hั̈тсH, M.J. \& Plamann, T. (2001). Imaging conditions for reliable measurement of displacement and strain in high-resolution electron microscopy. Ultramicroscopy 87 (4), 199-212. 
Jouneau, P.H., Tardot, A., Feuillet, G., Mariette, H. \& CiBERT, J. (1994). Strain mapping of ultrathin epitaxial ZnTe and MnTe layers embedded in CdTe. J Appl Phys 75(11), 7310-7316.

Кoch, C.T., Özdöl, V.B. \& van Aken, P.A. (2010). An efficient, simple, and precise way to map strain with nanometer resolution in semiconductor devices. Appl Phys Lett 96 (9), 091901.

Liu, J.P., Li, K., Pandey, S.M., Benistant, F.L., See, A., Zhou, M.S., Hsia, L.C., Schampers, R. \& Klenov, D.O. (2008). Strain relaxation in transistor channels with embedded epitaxial silicon germanium source/drain. Appl Phys Lett 93(22), 221912.

Müller, K., Schowalter, M., Rosenauer, A., Rubel, O. \& Volz, K. (2010). Effect of bonding and static atomic displacements on composition quantification in $\operatorname{In}_{x} \mathrm{Ga}_{1-x} \mathrm{~N}_{y} \mathrm{As}_{1-y}$. Phys Rev $B$ 81(7), 075315.

Ploessl, R., Chapman, J.N., Thompson, A.M., Zweck, J. \& HoffMANN, H. (1993). Investigation of the micromagnetic structure of cross-tie walls in permalloy. J Appl Phys 73(5), 2447-2452.

PrewitT, J.M.S. (1970). Object Enhancement and Extraction, pp. 75149. New York: Academic Press.

Robertson, M.D., Corbett, J.M., Webb, J.B., Jagger, J. \& CurRIE, J.E. (1995). Elastic strain determination in semiconductor epitaxial layers by HREM. Micron 26(6), 521-537. (Papers presented at the 22nd Annual Meeting of the Microscopical Society of Canada/Societe de Microscopie du Canada.)

Rosenauer, A., Fischer, U., Gerthsen, D. \& Förster, A. (1998). Composition evaluation by lattice fringe analysis. Ultramicroscopy 72, 121-133.

Rosenauer, A., Gerthsen, D., Dyck, D.V., Arzberger, M., Böhm, G. \& Abstreiter, G. (2001). Quantification of segregation and mass transport in $\mathrm{In}_{x} \mathrm{Ga}_{1-x}$ As/GaAs Stranski-Krastanow layers. Phys Rev B 64(24), 245334.
Rosenauer, A., Gerthsen, D. \& Potin, V. (2006). Strain state analysis of InGaN/GaN sources of error and optimized imaging conditions. Phys Status Solidi A 203(1), 176-184.

Rosenauer, A., Gries, K., Müller, K., Pretorius, A., Schowalter, M., Avramescu, A., Engl, K. \& Lutgen, S. (2009). Measurement of specimen thickness and composition in $\mathrm{Al}_{x} \mathrm{Ga}_{1-x} \mathrm{~N} / \mathrm{GaN}$ using high-angle annular dark field images. Ultramicroscopy 109(9), 1171-1182.

Rosenauer, A. \& Schowalter, M. (2007). STEMSIM-A new software tool for simulation of STEM HAADF Z-contrast imaging. In Springer Proceedings in Physics, Vol. 120, Cullis, A.G. \& Midgley, P.A. (Eds.), pp. 169-172. Berlin: Springer.

Rosenauer, A., Schowalter, M., Glas, F. \& Lamoen, D. (2005). First-principles calculations of 002 structure factors for electron scattering in strained $\operatorname{In}_{x} \mathrm{Ga}_{1-x}$ As. Phys Rev B 72, 085326.

Tillmann, K., Lentzen, M. \& Rosenfeld, R. (2000). Impact of column bending in high-resolution transmission electron microscopy on the strain evaluation of GaAs/InAs/GaAs heterostructures. Ultramicroscopy 83(1-2), 111-128.

Uesugi, F., Hokazono, A. \& Takeno, S. (2011). Evaluation of two-dimensional strain distribution by STEM/NBD. Ultramicroscopy 111(8), 995-998.

Usuda, K., Numata, T. \& Takagi, S. (2005). Strain evaluation of strained-Si layers on SiGe by the nano-beam electron diffraction (NBD) method. Mater Sci Semicond Process 8(1-3), 155-159.

WALLER, I. (1927). Die Einwirkung der Wärmebewegung der Kristallatome auf Intensität, Lage und Schärfe der Röntgenspektrallinien. Ann Phys 83, 153-183.

Yu, W. \& Mader, W. (2010). Displacement field measurement of metal sub-lattice in inversion domains of indium-doped zinc oxide. Ultramicroscopy 110(5), 411-417. 